\section{X-Ray Circular Magnetic Dichroism}

\author{
G. Schütz, P. Fischer \\ Institute of Physics, University of Augsburg
}

\begin{abstract}
There is a growing demand for circularly polarized $\mathrm{x}$-rays at high-brilliance synchrotron-based sources since they allow the extension of XMCD and other spectroscopic and crystallographic techniques involving core-level absorption to their magnetic counterparts to provide detailed element- and symmetry-selective information about solids.
\end{abstract}

With the advent of very bright photon sources having a high degree of polarization, a variety of new methods has been developed to investigate magnetism in solids using $\mathrm{x}$-rays. One technique which is attracting steadily growing interest studies $\mathrm{x}$-ray magnetic circular dichroism

(XMCD) in core-level absorption by measuring the dependence, on the magnetization of a specimen, of the absorption coefficient of circularly polarized radiation in the vicinity of an absorption edge. The interest arises because dichroic effects can provide element- and symmetry-selective information about the polarization of electronic states at the Fermi level, and about local magnetic moments separated into spin and orbital contributions.

A variety of different types of experiments have been performed in the hard and soft $\mathrm{X}$-ray ranges since XMCD was discovered in 1985 at the Hamburger Synchrotron Radiation Laboratory (HASYLAB) at DESY, Hamburg [1]. The most studied systems involve absorption at the $\mathrm{L}_{2}$ - and $\mathrm{L}_{3}$ - edges of ferromagnetic $3 \mathrm{~d}$ transition metals [2] owing to the very large magnetic cross-sections.

The versatility and elegance of XMCD for solving problems in magnetism will be demonstrated by showing that:

a) a high sensitivity to local magnetic moments combined with element selectivity and the ability to separate spin and orbital magnet moments can be used to probe nanoscale structures such as technically important multilayer systems;

Gisela Schütz was appointed head of Experimentalphysik ॥ an der Mathematisch-Naturwissenschaftlichen Fakultät der Universität Augsburg, Memmingerstr. 6, D-86135 Augsburg, in 1993, having been appointed professor earlier in the year. She studied at the TU Munich where she received her PhD in 1984 and her Habilation in 1992. Professor Schütz was awarded the Otto Klung Prize in 1989.

Peter Fischer joined the University of Augsburg in 1993 as a research assistant after studying at the TU Munich, from which he received his PhD in 1994. b) methods used in spectroscopy or crystallography involving core-level absorption can be extended to their magnetic tributions in the extended $\mathrm{x}$-ray absorption fine structure beyond the near-edge range (so-called magnetic or spinpolarized EXAFS - SPEXAFS) can provide new information about short-range magnetic structure. In particular, it is shown that magnetic backscattering by $3 \mathrm{~d}$-metals is solely determined by the outermost spin-polarized d-shell and is unaffected by the angular momentum of the backscattering atom.

\section{Multilayer Systems}

Magnetic exchange phenomena arise when non-magnetic metal spacer layers are placed between magnetic layers. The nature of the coupling between ferromagnetic layers through the spacer is of particular interest because it determines

Fig. 1. Induced magnetic moments in a 13 Pt monolayer (ML)/2 Co ML system. a: Theoretically predicted distribution; $\mathbf{b}$ : distribution inferred from XMCD results obtained using $2 \mathrm{Co} \mathrm{ML} / 5 \mathrm{Pt} \mathrm{ML} / 3$ Ir ML/5 Pt ML/2 Co $M L$ repeated 30 times; $c$ : Experimentally determined absorption profiles for the $\mathrm{Co} / \mathrm{Pt} / \mathrm{lr}$ structure at the $\mathrm{Pt} L_{2}$ and $L_{3}$-edges and the corresponding dichroic signal $\Delta \mu$ (the spin and orbital moments $\mu_{L}$ and $\mu_{s}$ deduced by sum rules are given as inserts).

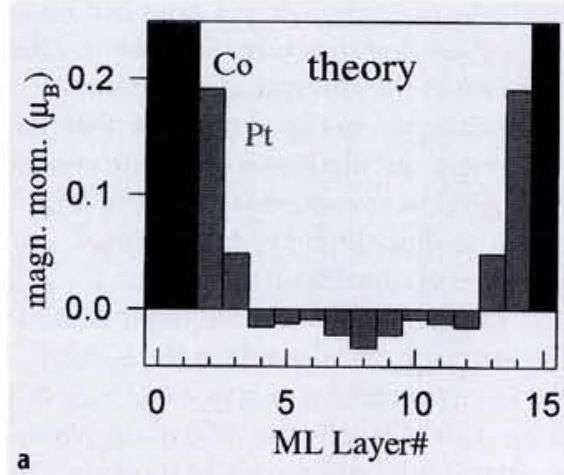
counterparts. For instance, dichroic con- macroscopic magnetic properties which are technological important. A high sensitivity to local magnetic moments combined with element selectivity means that these multilayer systems are very appropriate for XMCD studies. The technique also offers a unique new possibility for studying magnetism on an atomic scale because it can distinguish spin and orbital moments by comparing the dichroic signal for both initial state spin-orbit partners.

Fig. 1a gives the theoretically predicted distribution of the induced $\mathrm{Pt}$ moment for system composed of two monolayers (ML) of Co sandwiched between 13 monolayers of $\mathrm{Pt}$, as calculated by $\mathrm{H}$. Ebert. It is anticipated that exchange effects will induce polarization in the $\mathrm{Pt}$ spacer with an antiparallel coupling of the innermost layers with respect to the Co moments.
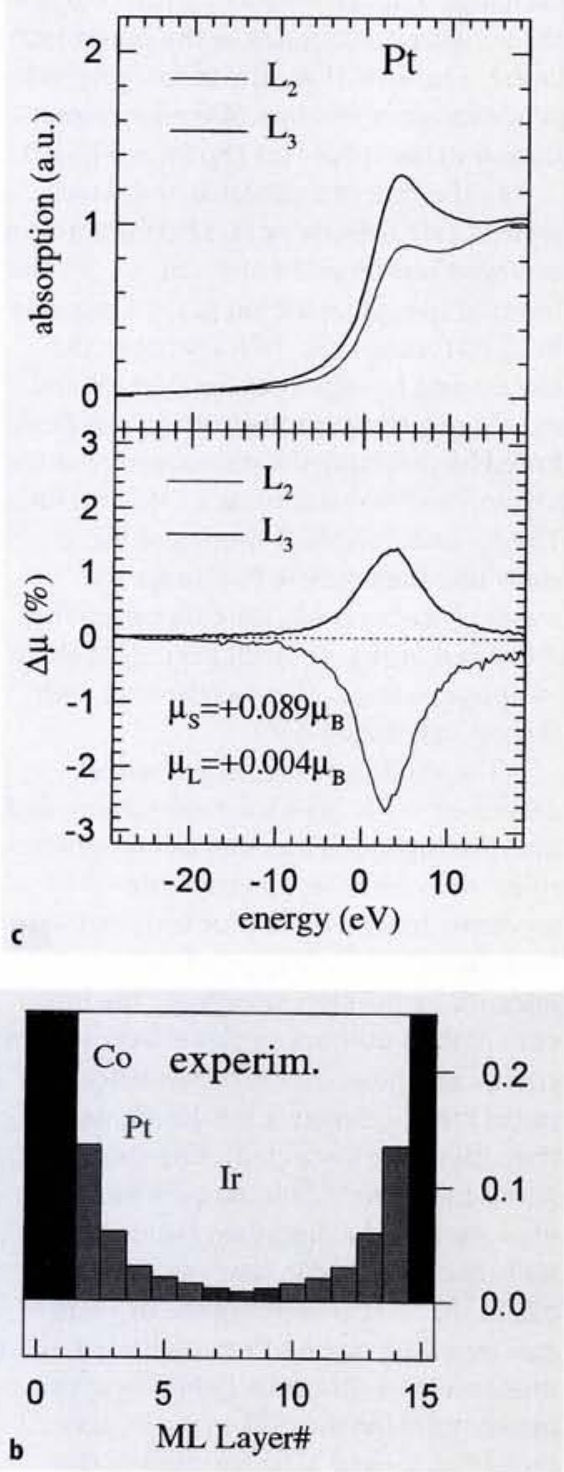
It is well known [1] that in the vicinity of ferromagnetic $3 \mathrm{~d}$-atoms $\mathrm{Pt}$ and Ir behave very similarly with respect to their induced magnetic moments. So it is expected that polarization effects in the $\mathrm{Co} / \mathrm{Pt}$ multilayered system will not change significantly if the three innermost $\mathrm{Pt}$ layers are replaced by Ir. XMCD studies at the $\mathrm{Ir}$ and $\mathrm{Pt} L_{2}$ - and $L_{3}$-absorption edges with a spin-orbit splitting of about $1.6 \mathrm{keV}$ in the 11.5-13.5 keV $x$-ray energy range have been performed using a setup installed at the RÖMO II station at Berlin's HASYLAB (see Fig. 2). The dichroic spectra at the $L_{2}$ and $L_{3}$-edges of $\mathrm{Pt}$ and $\mathrm{Ir}$ in a structure comprising $2 \mathrm{Co} \mathrm{ML} / 5 \mathrm{Pt} \mathrm{ML} / 3 \mathrm{Ir} \mathrm{ML} / 5 \mathrm{Pt}$ ML repeated 30 times unambiguously demonstrate that $\mathrm{Ir}$ is polarized parallel to Pt and Co (Fig. 1b). The average spin $\mu_{S}$ and $\mu_{L}$ orbital moments for Pt and Ir found by applying sum rules (see insert) are indicated in the figure. The data confirms the expected occurrence of a long-range exchange induced magnetic order, but not the antiparallel coupling of the innermost layers. The XMCD studies in fact give evidence for a distribution of the Pt magnetization in the spacer (as shown in Fig. 1b).

In the case of a $5 \mathrm{Co} / 4 \mathrm{Cu}$ multilayer system, calculations by $\mathrm{H}$. Ebert predict an average $\mathrm{Cu} d$-moment of $+0.0137 \mu_{B}$ for the induced spin polarization in the $\mathrm{Cu}$ spacer in its ferromagnetic state. Owing to the low $L_{2}$ - and $L_{3}$-edge energies ( $952 \mathrm{eV}$ and $932 \mathrm{eV}$, respectively), absorption was monitored by detecting the photocurrent at the $\mathrm{SX}_{700 / \mathrm{III}}\left(\mathrm{PM}_{3}\right)$ station at BESSY, Berlin. The $L_{2}-$ and $L_{s}$-XMCD spectra of Fig. 3 show that the predicted value for the moment is observed. Moreover, even the measured orbital contribution $\mu_{L}$ of about $-0.0005 \mu_{B}$ is in excellent agreement with theoretical expectations.

The validity of the simple model, described in the insert, for calculating and interpreting spectra as well as the sum rules for estimating the magnetic moments remain open problems owing to the relatively drastic approximations (for instance, multi-electron effects, the influence of final state spin-orbit effects and the energy and polarization dependence of radial matrix elements for the allowed transitions are neglected). The model also seems to be limited to cases for which the final state can be described either as a well-localized atomic state (as, for example, in the final $4 \mathrm{f}$-states in the $M_{4}$ - and $M_{5}$ spectra of the rare earth elements) or as an itinerant band-like state (which is approximately valid for the d-like final states excited in $L_{2}$ - and $L_{2}$-absorption in the
Fig. 2. X-ray magnetic circular dichroism using the very simple and effective inclined view transmission method. A large degree $(\approx 80 \%)$ of circular polarization is achieved, with a reduction of intensity relative to its maximum in the orbital plane to typically $60-80 \%$, by viewing the synchroton beam of energy $E$ at small angles normal to the plane of the electron orbit.

After monochromatization in a crystal monochromator, the incident $I_{0}$ and transmitted intensities $I(E)$ are monitored by ionization chambers. $I_{0} /(E)$ is related to the absorption coefficient $\left.\mu(E)=\ln \left\{I_{0} / /(E)\right) /\right\} \times$ for a sample thickness $x$, which is typically chosen to be several microns for $x$-rays with an energy $E$ larger than 5 $\mathrm{keV}$. The sample's magnetization is reversed periodically by reversing the field of the solenoid in which it is mounted. The dichroic signal corresponds to the difference $\Delta \mu=\mu^{+}-\mu^{-}$for the external magnetic field arranged parallel and antiparallel to the photon "spin" direction or helicity.

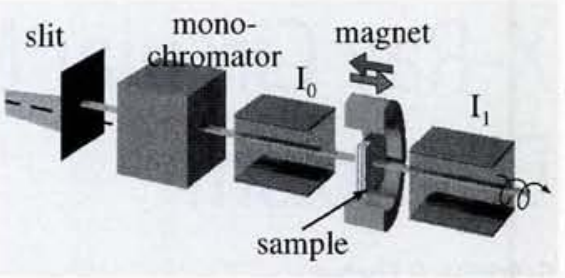

Other methods to measure the absorption may be more appropriate in the case of thicker samples, $x$-ray opaque substrates or softer $x$-rays. For instance, the detection of characteristic fluorescent light is well suited for very dilute components of thin layers, where probing depths of some tenths of a micron are needed. Monitoring the sample's photocurrent while extracting secondary electrons with mean-free paths of some nanometers using an applied voltage is extremely surface sensitive.
Fig. 3. XMCD in a $\mathrm{CO} M L / 4 \mathrm{Cu}$ multilayer system. $L_{2}$-and $L_{3}$ absorption profiles for $\mathrm{Co}(\mathrm{a})$ and $\mathrm{Cu}$ (b) and the corresponding dichroic profiles (c, d). The deduced spin and orbital moments are given as inserts.

heavier 3d-, 4d- and 5d-transition metals). The model therefore fails at the $L_{2}$ - and $L_{3}$ edges of the rare earths because the final state is neither pure atomic nor pure bandlike in character. For atoms such as Mn with atomic numbers below 25 , the $L_{2}-$ and $L_{3}$-states are not well resolved experimentally so $\mu_{L}$ can only be estimated.

\section{Spin-polarized EXAFS}

Measurements of $x$-ray magnetic circular dichroism made at the $L$-edges of rare-earth elements demonstrate the presence of dichroic contributions even beyond the near-edge range, i.e., in the magnetic or spin-polarized extended x-ray absorption fine structure (SPEXAFS). This is shown in the absorption spectrum for Gd metal given in Fig. 4. It is clear that the "magnetic" oscillations in this pure system have a frequency which is similar in magnitude to those found in conventional, i.e., spin-averaged, structures.

The ratio of the relative amplitudes of the magnetic contribution at the $L_{2}$ - and the $L_{3}$-edges corresponds to about 0.02 to 0.04 . This is exactly the ratio of the photoelectron polarization $\left\langle\sigma_{z}\right\rangle$ so it points directly to the origin of these dichroic features: the EXAFS result from interference between the outgoing electron wave with a wave number $k$ and the incoming waves backscattered by the nearest neighbours located at a distance $r$, as illustrated in Fig. 5. The frequency of the EXAFS is thus given by the $\sin (2 k \cdot r)$ Bragg scattering law. Spin-polarized EXAFS can even be observed at $K$-edges, but since $\left\langle\sigma_{z}\right\rangle$ is only 0.03 the amplitudes observed are an order of magnitude smaller than those arising at $L$-edges.

As discussed in the insert, the outgoing photoelectron wave created by absorption of circularly polarized $\mathrm{x}$-rays is spinpolarized. If the magnetic spin-moment of the neighbouring atom, i.e., the sum of the spins of its magnetic electrons, is also polarized, an exchange contribution in addition to the Coulomb scattering potential takes part in the scattering process. This results in a magnetic contribution to the backscattering amplitude. So when the backscattering neighbour is also spin polarized, the backscattering amplitude will be different for parallel and antiparallel spin orientations of the photoelectron 


\section{The Vector Coupling Model}

The physical origin of XMCD and its global characteristics can be discussed in the frame of a simple vector coupling model [1]. The model is based on the dipole approximation for the photoelectron transition and the constraint that with a change $\Delta m_{l}$ in the orbital quantum number $m_{l}$ equal to $+(-)_{1}$ for the absorption of a right- (left-) handed photon, the photoelectron exits with a mean spin polarization $\left\langle\sigma_{z}\right\rangle$ in the photon beam direction $z$. Hence, for transitions from initial $p_{1 / 2}$ and $p_{3 / 2}$ spin-orbit states into a d-like final state, $\left\langle\sigma_{z}\right\rangle$ amounts to $-1 / 2$ and $+1 / 4$, respectively. The radial matrix elements for allowed dipole transitions into final s-states are about two orders of magnitude weaker and can therefore be neglected.

For very low photoelectron energies $(E<$ $20 \mathrm{eV}$ ) and itinerant final states the absorption coefficient is described by Fermi's Golden Rule, i.e., $\mu_{l}(E) \approx|M(E)|^{2} \rho_{l}(E)$, where $\rho_{l}(E)$ reflects the density of states (DOS) of the unoccupied bands with a defined angular momentum $l$ near the Fermi level and $M$ is the transition matrix element for a photon with energy $E$. The final states show exchange splitting if the absorbing atom has a magnetic spin moment. The majority (minority) band $\rho^{+}\left(\rho^{-}\right)$ is shifted below (above) the Fermi level, as illustrated in the figure for the (5)d-states of $\mathrm{Pt}$ polarized by ferromagnetic Fe neighbours. A local magnetic spin moment is therefore induced. Its magnitude corresponds to the integrated difference $\mu_{1} / \mu_{B}=\left\{\left\{\rho^{+}(\mathrm{E})-\rho^{-}(\mathrm{E})\right\} \mathrm{d} E\right.$ where $\mu_{B}$ is the permeability of vacuum.

For the case of absorption of a right circularly polarized photon at the $L_{2}$-edge in $\mathrm{Pt}$, a $\left\langle\sigma_{z}>=-1 / 2\right.$ means that three times more photoelectrons with spin antiparallel to the photon propagation direction $z$ are ejected than electrons with spin parallel. Owing to Pauli's exclusion principle, the photoelectrons can be considered as a probe for the final-state spin polarization projected onto the photon reciprocal lattice $k$-vector.

An applied external magnetic field aligns the Pt magnetic moment, i.e., the spin of the majority electrons are parallel or antiparallel to the $z$ direction. Following Fermi's Golden Rule, the absorption coefficient $\mu$ for the situation depicted is larger than the coefficient $\mu^{+}$ with the reversed magnetization (see Fig. I). The difference in the absorption coefficients $\Delta \mu \approx|M(E)|^{2}<\sigma_{z}>\Delta \rho$ scales with the spin density distribution of the unoccupied bands. In analogy to the local spin moment given by $\mu_{s} / \mu_{B}=\int^{E_{f}} \Delta \rho^{\text {occupied }} \mathrm{d} E$, the "hole moment" can be defined as the integral $\int_{f}^{E} \Delta \rho^{\text {occupied }} \mathrm{d} E$. It has the same value as the actual moment, but opposite sign, provided the occupied and unoccupied DOS contain all ten 5 d electrons. Thus the integrated dichroic signal measures directly the local spin moment.

The predicted absorption profile in the vicinity of $L_{2}$ - and $L_{3}$-edges for an atom with an unfilled d-shell is sketched schematically in the Fig. IIa. The high density of states results in the resonantly enhanced absorption thresh-

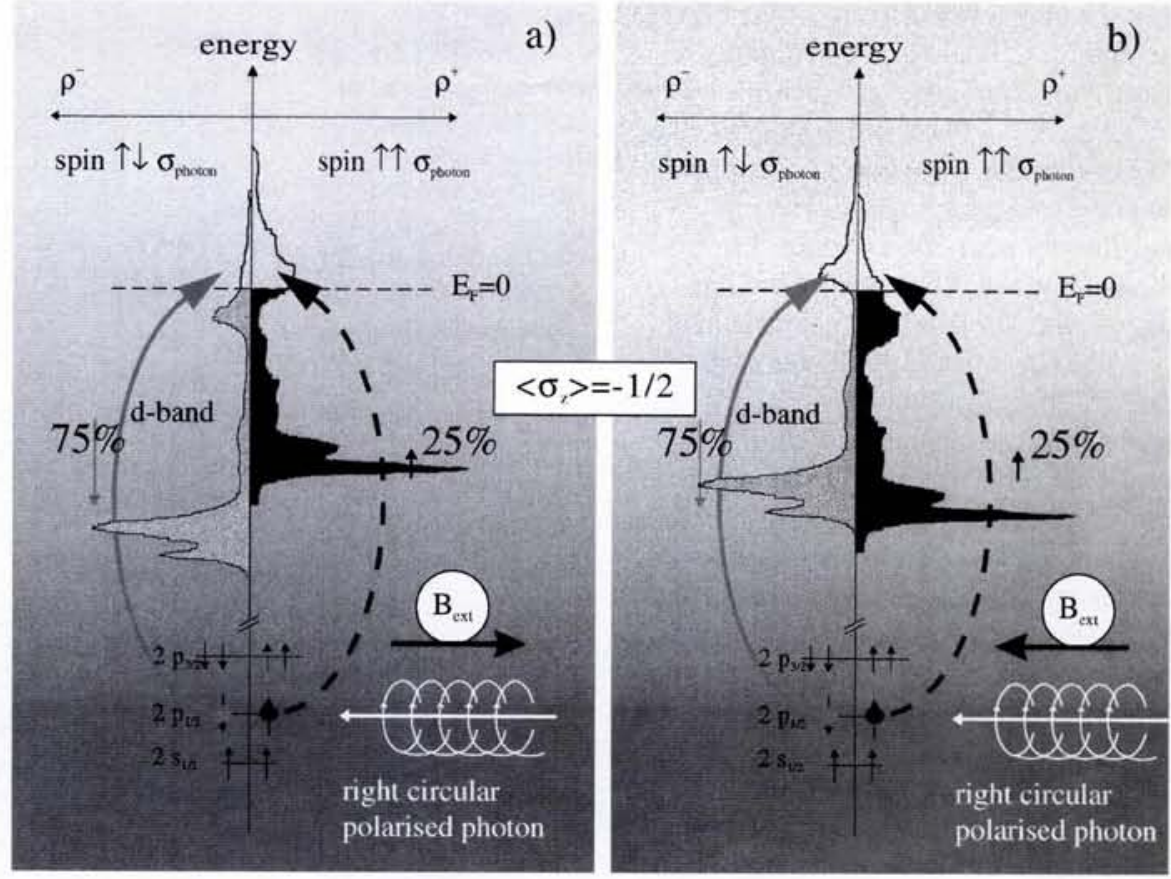

Fig. I: The photoelectron transition in the $L_{2}$-absorption of a right circularly polarized photon at a Pt impurity in a ferromagnetic Fe host. The d-band at the Fermi energy is exchange split owing to the formation of a local magnetic spin moment. The spin orientation of the majority-like electron relative to the photon helicity is flipped by reversing the external magnetic field along the photon propagation direction (see $\mathbf{a}$ and $\mathbf{b}$, respectively).

Fig. II: Schematic $L_{3,2}$-absorption profile (a) for an absorbing atom with unfilled d-shell. b: Corresponding XMCD signal for a complete spin polarization of the empty final d-states and zero orbital polarization. c: XMCD profile for complete final-state orbital polarization and vanishing spin density.

old. If the local spin moment is aligned antiparallel to the photon beam direction, i.e., the final spin DOS $\Delta \rho^{\text {occupied }}$ is on average aligned parallel, then the dichroic signal is negative (positive) at the $L_{2}\left(L_{3}\right)$-edge owing to the positive (negative) value of $\left\langle\sigma_{z}\right\rangle$. Since the transition probability is twice as large at the $L_{3}$-edge, the photoelectron spin polarization vanishes for zero or experimentally non-resolvable initial spin-orbit states.

However, this analysis neglects the fact that due to the constraint $\Delta m_{l}=+1$ (for righthanded photons) the outgoing photoelectron also has a finite orbital polarization $\left\langle l_{z}\right\rangle$ that depends only on the symmetry of the initial state. Thus, for example, at both $L_{2}$-and $L_{3}$ edges, $\left\langle l_{z}\right\rangle=+3 / 4$ if the total orbital polarization in the $L=2$ final state is defined as $\left\langle l_{z}\right\rangle=$ 1. According to this simple model, which readily explains the sensitivity of the XMCD signal to the spin moment, the outgoing photoelectron also probes the local orbital moment. In the (unrealistic) case that an orbital moment oriented antiparallel to the photon helicity is only present one would expect a dichroic profile of the type shown in Fig.IIc.

If in the (realistic) case the absorbing atom carries a magnetic moment with both spin and orbital contributions $S$ and $L$, the strength of the magnetic absorption should increase (decrease) at the $L_{3}$-edge at the expense of adsorption at the $L_{2}$-edge for parallel (antiparallel) coupling of $L$ and $S$ depend-

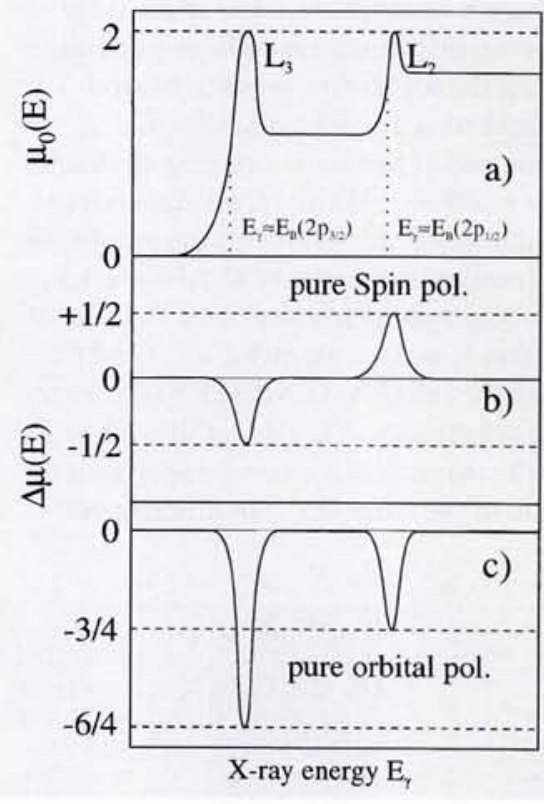

ing on their relative magnitudes. This picture describes the application of Thole and Carra's sum rules where the intregal $\int L_{2}+L \Delta \mu(E) \mathrm{d} E$ scales with the orbital momentum and $\int L_{2}+$ $L_{3}\left\{2 \Delta \mu(E)_{L_{2}}-\Delta \mu(E)_{L_{3}}\right\} \mathrm{d} E$ with the spin momentum [3].

Interpretating XMCD at $K$-absorption edges for an initial s-state is complicated since a very small photoelectron spin polarization (of the order of $10^{-2}$ ) is induced by spin-orbit splitting of the final state, while the orbital polarization is $100 \%$. So correlating the magnetic absorption profile to the spin and orbital moments of the p-like final states is complex and depends strongly on spin-orbit effects. 
and the magnetic electrons of the nearest neighbour (so-called singlet and triplet scattering). Moreover, the dichroic effect in the EXAFS will change sign if the relative orientation of the spin of the photoelectron and the spin of the magnetic neighbours is reversed, either by inverting the photon helicity or by flipping the direction of the external magnetic field.

The effect of the difference of the exchange interaction between singlet and triplet scattering is introduced in the conventional EXAFS formula by an additive contribution scaling with $\left\langle\sigma_{z}\right\rangle$ to the Coulomb interaction parameters. The backscattering amplitude $F_{0}$ has to be replaced by $F=F_{o} \pm\left\langle\sigma_{z}\right\rangle F_{c}$, where $F_{c}$ is the magnetic backscattering amplitude, with the corresponding phase shift $\varphi=\varphi_{0} \pm\left\langle\sigma_{z}>\varphi_{c}\right.$.

\section{Spin-polarized Backscattering}

The electronic neighbour is identical to the magnetic one in elementary ferromagnetic systems. Consequently, Fourier transforms of EXAFS and of its magnetic counterpart are nearly identical in these systems [4]. Furthermore, it has been shown that the relative strengths of the lines in the transforms, whose positions mark the separation between nearest neighbours, are related to the spin moments of the backscattering atoms. So it is possible to deduce the $k$-dependence of the magnetic backscattering amplitude $F_{c}$ from experimental SPEXAFS spectra.

Fig. 6 compares experimentally determined $F_{\mathrm{c}}$ spectra for metallic $\mathrm{Co}$ and $\mathrm{Ni}$ with the calculated Coulomb backscattering amplitudes. It is evident that the ratio of the maxima of $F_{c}$ scale directly with the ratio of the magnetic spin moment per
Fig. 4. XMCD absorption spectrum at the $G d$ metal $L_{2}$-and $L_{3}$-edges with no background subtraction. The EXAFS structures beyond the absorption edges are also magnified in amplitude by factors of 10 and 20 at the $L_{2}$-and $L_{3}$ edges, respectively.
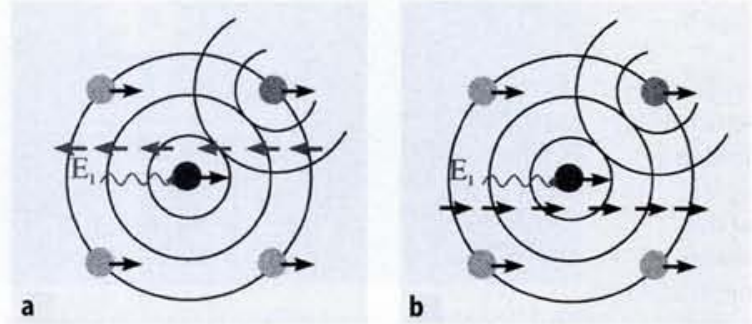

Fig. 5. The physical origin of magnetic EXAFS. After absorption of a polarized photon of energy $E$, the photoelectron wave is spinpolarized in the photon propagation direction. If the backscattering neighbour is also spin polarized the backscattering amplitude is different for singlet (a) and triplet (b) scattering. atom. In contrast to the $F_{0}$ distribution which is extended to higher $k$-values, the magnetic contribution is concentrated in a region between $2.5 \AA^{-1}$ and $7.5 \AA^{-1}$. A comparison of the backscattering amplitude within the whole $3 \mathrm{~d}$ series shows that it is in exactly this $k$-range that 3 d-electrons make the predominant contribution to the scattering potential. Thus the different $F_{c}$ distributions in $3 \mathrm{~d}$-metals can be

explained solely in terms of backscattering from the outermost spin-polarized d-shell.

An important question now arises: does the existence of an angular momentum in the absorbing and/or backscattering atom influence the SPEXAFS ? To

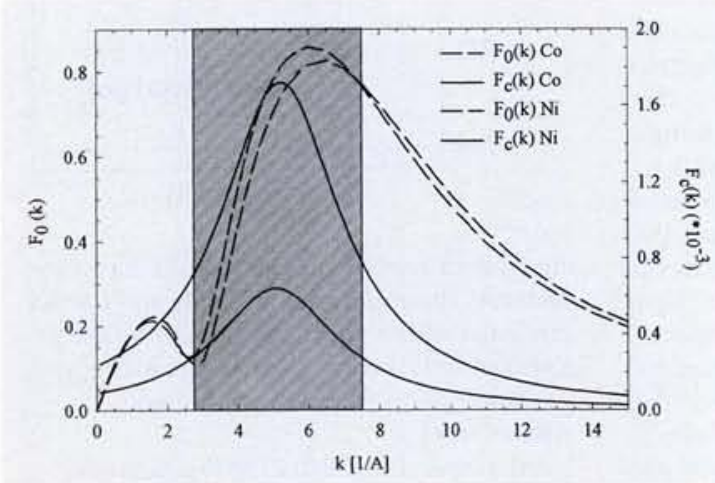

Fig. 6. A comparison of the calculated Coulomb backscattering amplitudes $F_{0}$ for the $3 \mathrm{~d}$-transition metals $\mathrm{Co}_{0}$ and Ni with the magnetic backscattering amplitude $F_{c}$. The results are plotted as a function of the reciprocal lattice vector $k_{;}$. $F_{0}$ and $F_{\text {c }}$ were deduced from the experimental $K$-edge spin-polarized EXAFS. The shaded area marks the region where $3 \mathrm{~d}$-electrons make the predominant contribution to the backscattering amplitude.
Fig. 7. The Fourier transforms of the $L_{2}$ and $L_{3}$-edge EXAFS of $\mathrm{Ho}_{0}$ in $\mathrm{H}_{3} \mathrm{Fe}_{5} \mathrm{O}_{12}$ (dotted curve; left-hand scale) compared with the Fourier transforms of the SPEXAFS magnetic contribution $\Delta \mu(k)$ to the absorption (solid curve; right-hand scale). The oxygen nearest neighbours are non-magnetic so their contribution vanishes in the SPEXAFS.

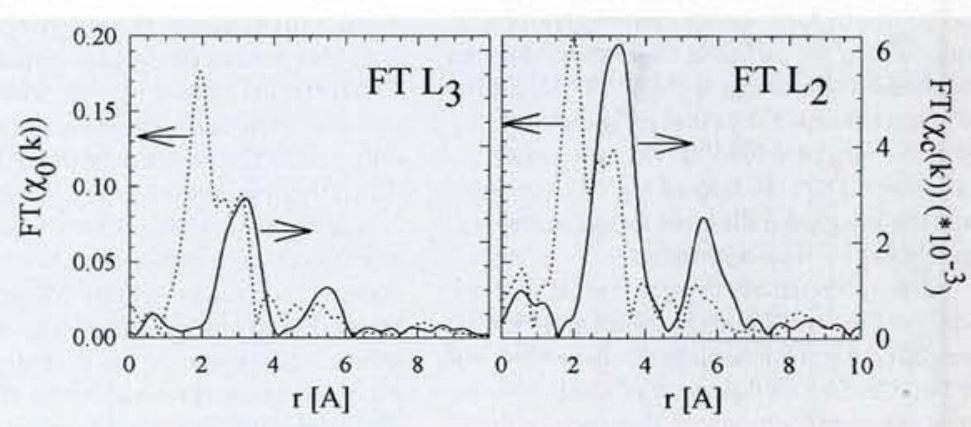

address this problem one can use the garnet $\mathrm{H}_{3} \mathrm{Fe}_{5} \mathrm{O}_{12}$, where according to Hund's rules the $\mathrm{Ho}_{4} \mathrm{f}$-core has an orbital moment $L=6$ and a spin moment $S=2$ coupling parallel to the orbital moment.

Fig. 7 compares Fourier transforms for $\mathrm{Ho}_{2}$ - and $L_{3}$-EXAFS in $\mathrm{H}_{3} \mathrm{Fe}_{5} \mathrm{O}_{12}$ with the oxide's spin-resolved EXAFS transforms. As is typically the case for oxides, the EXAFS Fourier transforms are dominated by a strong first maximum resulting from backscattering at the oxygen nearest neighbours; the second peak indicates the position of the next (four) $\mathrm{Fe}$ neighbours. However, in the SPEXAFS transforms the prominent oxygen peaks have vanished at the expense of an increase in the maxima identifying the next and next-nearest group of unresolved coordination shells at a distance $r$ of $3 \AA$ consisting of $16 \mathrm{Fe}$ and $8 \mathrm{Ho}$ atoms. Fig. 7 therefore demonstrates that to within the statistical uncertainties, the ratio $L / S$ of the moments does not deviate from a value of -2 , even for the range centred on the unre- 
solved $16 \mathrm{Fe} / 8 \mathrm{Ho}$ coordination shells at 3 $\AA$ where the $L / S$ ratio for the nearest neighbours is on average close to 1 . So magnetic backscattering seems to be unaffected by the angular momentum of the backscattering atom.

\section{Conclusions}

It has been demonstrated that dichroic near-edge effects provide a powerful tool for probing the magnetic characteristics of unoccupied density of states and determining, in appropriate cases, local spin and orbital moments separately and with high accuracy. So X-Ray Magnetic Circular Dichroism allows one to study the local magnetic properties of ferro- and ferri-magnetic solids in an element- and symmetry-selective manner.

Moreover, the analysis of dichroic effects beyond the near-edge range (spinpolarized EXAFS) probes magnetic shortrange order and the magnetic moments of neighbouring atoms and allows a clear distinction between magnetic and nonmagnetic atomic neighbourhoods.

Every spectroscopic or crystallographic technique involving core-level absorption can be extended, in principle, to its magnetic counterpart. Indeed, photons with energies close to an absorption edge are already being applied in magnetic Anomalous Small Angle x-ray Scattering, atomic contrast techniques and $\mathrm{x}$-ray microscopy and microtomography. These innovations are made possible the development of high-brilliance $\mathrm{x}$-ray sources and they demonstrate the growing demand world-wide for circularly polarized $\mathrm{x}$-rays.

\section{Acknowledgments}

The authors wish to thank their colleagues, especially $\mathrm{H}$. Ebert, for support and collaboration.

\section{References}

[1] G. Schütz et al., Phys. Rev. Lett. 58 (1987) 737; G. Schütz, M. Knülle \& H. Ebert in Resonant Anomalous X-Ray Scattering: Theory and Applications; Eds: G. Materlik, C.J. Sparks \& K. Fischer (Elsevier Science, 1994) 535.

[2] C.T. Chen et al., Phys. Rev. B 42 (1990) 7262; F. Sette et al., Proc. 6th Int. Conf. on Xray Absorption Fine Structure, Ed: S.S. Hasnain (Ellis Horwood, 1991) 96.

[3] B.T. Thole et al., Phys. Rev. Lett. 68 (1992) 1943; P. Carra et al., Phys. Rev. Lett. 70 (1993) 694.

[4] G. Schütz, et al., Phys. Rev. Lett. 62 (22) (1989) 2620; G. Schütz \& D. Ahlers, in SpinOrbit Influenced Spectroscopies of Magnetic Solid; Eds: H. Ebert, G. Schütz, Lecture Notes in Physics 466 (Springer, 1996).

\section{Large Bose Condensate Imaged}

The highlight of the 15th General Conference of the EPS Condensed Matter Division: EPS-CMD'96 (Stresa; 22-25 April) was an invited plenary talk by Wolfgang Ketterle from MIT who described major advances in producing and probing a Bose-Einstein condensate (BEC). His group has produced a condensate of 5 million atoms, 10-times greater than the previous record it set last year. Most importantly, the group has been able to directly observe the condensate in the atom trap, and not just the fragments of an exploding condensate as was done previously.

The key development is a novel trap. Like previous devices, trapping uses a strong, inhomogeneous magnetic field but without a zero magnetic field near the centre of the trap, where atoms experiencing this field loose their (spin) orientation and escape. The experiments that achieved BEC last year used a rotating field or optical forces to push the atoms to locations where the magnetic field was non-zero. Such tricks are no longer necessary, because the new trap uses DC magnetic fields which have a minimum value of 1 Gauss and increase in all directions from the centre. The group has also come up with a novel "cloverleaf" winding pattern which gives very good optical access - a crucial requirement because 11 laser beams are needed for cooling and probing the atoms. Trapped atoms suffered very little heating - a condensate could be kept for more than $20 \mathrm{~s}$, and the evaporative cooling was more efficient than before resulting in the large condensate of $\mathrm{Na}$ atoms.

With the sizable condensate, the group has tested theoretical predictions, some dating back to the 1940 s. Measurements on the condensate agree with theoretical predictions that the fraction of atoms in the condensate should vary with temperature $T$ as 1- $\left(T / T_{c}\right)^{3}$, where $T_{c}$ is the critical temperature at which the condensate forms. Predictions on the amount of repulsive energy between the $\mathrm{Na}$ atoms in the condensate have also been verified. The condensate was pencil-shaped (about $150 \mu \mathrm{m}$ long and $8 \mu \mathrm{m}$ wide).

It was also large enough to be directly observed for the first time using laser light scattered onto a sensitive camera. A direct image of an atomic matter wave with a half-wavelength of $150 \mu \mathrm{m}$ was obtained. A thermal gas with such a long deBroglie wavelength would be at a temperature of 1.5 picokelvin.

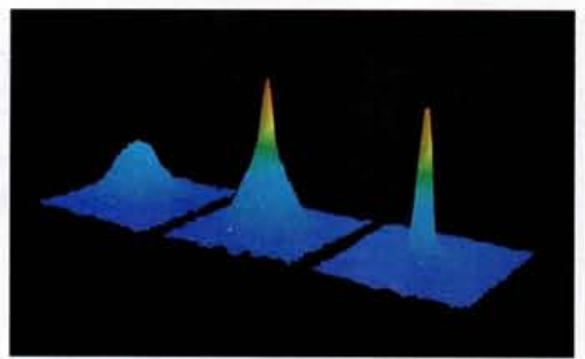

A direct image of an expanding Bose-Einstein condensate showing the strong asymmetry in the expansion owing to repulsion between the condensed atoms. A characteristic feature of the trap developed by Ketterle and coworkers at MIT is the extreme aspect ratio of the condensate ( $150 \mu \mathrm{m}$ long by $8 \mu \mathrm{m}$ wide), which can seen in the expansion. The images show that the expanding cloud has both a condensed part and a normal part. The latter expands isotropically like a classical gas whereas the condensate expands into an elliptical shape. The width of the field of view is $6 \mathrm{~mm}$. In the early phase, the cloud appears larger than the true size owing to complete absorption of the laser probe light.

The group has also developed a nondestructive imaging technique. The absorption of near-resonant light and reemission in random directions which was used previously caused the atoms to recoil, since light was absorbed, and the recoil energy heated up the condensate and destroyed it. The new imaging technique employs the dispersion of $\mathrm{x}$-rays: the atoms in the condensate deflect the $\mathrm{x}$ rays at a small angle. The recoil of the atoms is small, and can even be transferred to the magnetic trap through the Mössbauer effect. So the condensate can be imaged without heating to obtain spatially resolved images. Two images of the same condensate have been taken, and multiple images seem possible - a technique that should open the door to studies of the dynamics of a single condensate.

Finally, the group has taken the first step in probing the condensate's optical properties, one of the mysteries of this form of matter. By shinning photons on the condensate with a frequency $1.7 \mathrm{GHz}$ away from the resonance frequency of the condensate it was found that a sodium condensate acts as a lens: indeed, it is transparent but deflects light at a small angle. The angular distribution of the scattered light is anisotropic.

Now that there are several ways to probe condensates one can address specific aspects (e.g., superfluidity; the nature of excited states) of the macroscopic physics of Bose-Einstein condensate "superatoms" consisting of 5 million atoms. Interference effects between two superatoms can perhaps be observed by cutting the condensate into two parts using a laser. 